\title{
Factors associated with Toxoplasma gondii infection in confined farrow-to-finish pig herds in western France: an exploratory study in 60 herds
}

V. Djokic ${ }^{1,4+}$, C. Fablet $^{2+}$, R. Blaga ${ }^{1 \dagger}$, N. Rose ${ }^{2}$, C. Perret $^{3}$, O. Djurkovic-Djakovic ${ }^{4}$, P. Boireau ${ }^{3}$ and B. Durand ${ }^{5^{*}}$ (D)

\begin{abstract}
Background: Infection by Toxoplasma gondii postnatally can occur after ingestion of contaminated meat or water (tissue cysts/oocysts). In Europe, percentage of meat borne infections is estimated between 30 and 63 \%, out of which pork makes the most important source. The aim of this study was to (i) investigate the seroprevalence of $T$. gondii in intensive pig farms from western France; and (ii) identify the risk factors associated with seropositivity.

Methods: Data were collected between November 2006 and February 2008 in 60 intensive farrow-to-finish farms, where sera were taken from 3595 fattening pigs, weaned and suckling piglets. Information about three classes of potential seropositivity risk factors were obtained through a questionnaire concerning: (i) breeding characteristics; (ii) farm management; and (iii) husbandry and hygiene. The modified agglutination test (MAT) was used for detection of specific anti T. gondii antibodies in pig sera, starting from 1/6 dilution.

Results: The overall proportion of seropositive animals was $6.9 \%$, but the proportion of herds with at least one positive pig was $100 \%$. Multivariate logistic mixed model showed an increased seropositivity risk in weaned compared to suckling piglets, and a decreasing risk for mid-sized and large farms. The presence of a Danish entry facility, that clearly separates clean and dirty areas, had a protective effect on $T$. gondii seropositivity as well.

Conclusions: The observed proportion of herds with at least one T. gondii seropositive animal provides further evidence that even in confined conditions of pig breeding, infection occurs, and is common. The highest risk for acquiring $T$. gondii is at the end of weaning period. Smaller confined pig farms demonstrate higher $T$. gondii seropositivity levels. This study also showed that Danish entry on farm buildings provides effective protection against T. gondii.
\end{abstract}

Keywords: Toxoplasma gondii, Intensive pig farm, Risk factors

Abbreviations: $\mathrm{Cl}$, Confidence intervals; MAT, Modified agglutination test; OR, Odds-ratios; PCV, Porcine circo virus; PRRSV, Porcine reproductive and respiratory virus

\footnotetext{
* Correspondence: benoit.durand@anses.fr

tEqual contributors

${ }^{5}$ ANSES, Université Paris-Est, Laboratoire de santé animale de Maisons-Alfort

Epidemiology unit, Maisons-Alfort, France

Full list of author information is available at the end of the article
} 


\section{Background}

Toxoplasma gondii is one of the world's most widespread food borne pathogens. The parasite from phylum Apicomplexa is adapted for infection of all warm-blooded animals including humans. The characteristic of $T$. gondii is that parasites can be transmitted directly among intermediate hosts, including pigs, by predation or scavenging without need for the sexual part of the life-cycle in felids. The ingestion of food or water contaminated with different parasitic life stages (oocysts/tissue cysts) are the two major routes for postnatal transmission of $T$. gondii [1].

Infection in humans is generally benign, characterized by mild, flu-like symptoms. However, if maternal infection occurs in pregnancy, $T$. gondii may cause serious damage on the developing foetus; and in immunosuppressed individuals lesions of nervous system and eyes can occur with even life-threatening consequences [2]. Due to its zoonotic character, this parasitosis represents an important public health hazard.

The percentage of meat-borne infections of the total number of toxoplasmosis patients has been estimated between 30 and $63 \%$ in Europe [3]. Along with sheep, pigs are the species mostly associated with human $T$. gondii infection [4]. Nowadays three pig farming systems are recognized: (i) intensive, (ii) free-range (outdoor) and (iii) organic farming. In intensive farms animals are kept in strictly confined conditions, without any access to outside environment. Pigs are separated in categories, defined as batches (contemporary group of pigs), from where all animals are transported in the same time (all-in, all-out). In contrast, both organic and outdoor pigs have access to natural light, weather conditions and contact with other animal species. Mainly, organic pigs are bred in large open space pens without closed facilities, fed by seasonal food without chemical processing, and the system all-in, all-out is not practiced.

Various levels of seroprevalence have been reported during the last decade in European pigs and wild boars. In five European countries (the Netherlands, Sweden, France, Latvia and Switzerland), published estimates allow comparing seroprevalence levels observed in intensively bred pigs (the dominant breeding system in Europe), in organic/outdoor pigs and in wild boars. A common trend is observed in four of these five countries, with low seroprevalence levels in intensively bred pigs $(0.4 \%$ in the Netherlands [5], 1 \% in Sweden [6], $2.9 \%$ in France [7], and $0.4 \%$ in Latvia [8]), medium seroprevalence levels in organic/outdoor pigs (5.6\% in the Netherlands [5], $8 \%$ in Sweden [9], $6.3 \%$ in France [7], and $6.2 \%$ in Latvia [8]), and high seroprevalence levels observed in wild boars (24\% in the Netherlands [10], $50 \%$ in Sweden [9], $23 \%$ in France [11], and $33 \%$ in Latvia [8]). An opposite trend had been reported in Switzerland [12], with similar seroprevalence observed in intensively bred (14\%) and organic/ outdoor pigs (13\%), and a lower seroprevalence observed in wild boars $(7 \%)$. The similar trends and seroprevalence levels observed in the four above countries are coherent with the hypothesis that wild boar and organically/freeranged pigs get infected mainly from an oocyst-contamina ted environment by rooting, or by consumption of infected rodents, whereas pigs not exposed to oocyst-contaminated environment, nor infected rodents (i.e. pigs from confined, intensive farms) appear to be less often seropositive.

Routine $T$. gondii testing of pigs, destined for human consumption has not been introduced in any country [13]. Therefore the existence and location of endemic areas, the sources of pig infection, the frequency of infected pigs on the markets, and the role these animals play in human $T$. gondii epidemiology remain unknown.

In 2013, a French nation-wide study of $T$. gondii in intensively bred pigs slaughtered for human consumption was conducted. Samples from randomly selected piglets and fattening pigs were taken in 26 slaughterhouses. The resulting seroprevalence rates were $2.6 \%$ in piglets and $2.9 \%$ in fattening pigs [7]. However, the study design (based on samples taken in slaughterhouses) did not allow estimating the proportion of infected farms, or the proportion of seropositive animals in these farms.

Therefore, the aim of the present work was to analyse the presence of $T$. gondii in intensive pig farms and to investigate: (i) the farm-level and within farm proportion of seropositives, and (ii) factors associated with seropositivity. For this purpose, sera and data related to herd characteristics, biosecurity, management and housing conditions (obtained by questionnaire) were collected from 60 confined farrow-to-finish farms. All studied farms were located in western France, the region were almost $60 \%$ of total country's pork production is located $[14,15]$.

\section{Methods \\ Database and serum bank}

Sera samples and epidemiological data were obtained from a previous study, conducted from November 2006 to February 2008 in 125 farrow-to-finish pig productions from western France with more than 100 sows per farm [14]. In each of these 125 farms, blood samples were collected from pigs. A face-to-face interview, which included 610 questions, was conducted with the farmer, as well as herd investigations (using a standardized protocol with 257 measurements and observations, see Additional file 1 for the part of the questionnaire used for the present study), by the Swine epidemiology and welfare unit (ANSES), as described in [14]. The resulting dataset included a general description of the herd and of its neighbourhood, and data about biosecurity and hygiene practices, as well as management and animal husbandry. The serum bank included 
a total number of 7500 sera from pigs of 4 age classes: <1 month, $1-2$ months, $2-3$ months and $>3$ months.

\section{Herd selection and risk factors}

A written consent from farmers (represented by their organisations of producers) was asked for the use of pig sera and data in the present study. A farm was considered as T. gondii-positive if specific antibodies were found in at least one pig. The a priori estimate of the proportion of positive farms was set at $20 \%$. For a relative precision of $5 \%$, the sample size was calculated to be 60 farms, which were randomly chosen among 125 available from the database. Assuming a withinherd prevalence of $5 \%$, the number of samples per farm necessary to detect $T$. gondii presence (i.e. to obtain at least one positive serum) at a $95 \%$ confidence level was 60 sera. These 60 sera were equally distributed in the 4 age classes. Thus, for each of the 60 chosen farms, 60 pig sera were randomly selected in the serum bank: 15 sera from $<1$ month suckling piglets, 15 sera from 1 to 2 months weaned piglets, 15 sera from 2 to 3 months fattening pigs, and 15 sera from $>3$ months fattening pigs.

On all farms pigs were kept in completely confined conditions, without any outside access neither for piglets or sows. Farm buildings were separated according to each of the 4 age classes: farrowing rooms for suckling piglets (and sows) from birth to one month of age, weaning rooms for one to two months old piglets, fattening rooms for animals of $2-3$ months of age, and rooms for $>3$ months old animals.

The dataset associated to farm- and age-class specific serological results (number of tested and number of positive animals) included both room-level and farm-level variables. Room-level variables were the type of floor (slatted floor, straw, combination of slated and concrete floor) and the feed distribution method (automatic feeders or manual). Farm-level variables included hygiene practices: the use of specialized boots (yes/no) and clothes (yes/no), the compliance with all-in, all-out management (time in days during which pens/rooms/buildings were left empty in post-weaning and post-fattening processes), and the presence of a Danish entry facility (yes/no): a closed facility with at least two doors that open successively, upon entering the breeding rooms. All farms had controlled airflow, used pressurized steam for cleaning and specialized external company always performed rodent control; these elements were thus not considered in the analysis. Farm-level variables also included the number of sows (on a logarithmic scale), used as a proxy for herd size. Finally the presence of dairy cows (yes/no), beef cattle (yes/no), as well as sheep (yes/ no), goats (yes/no) and poultry (yes/no) breeding facilities on the same farm were considered.
A total number of 14 individual-level (1 variable), room-level (2 variables) and farm-level (11 variables) variables were thus considered (Table 1).

\section{Serology testing}

Detection of T. gondii IgG antibodies was performed on sera by MAT, as described by Halos et al. [16], since this is a species-independent serological test. MAT is considered as a gold standard for the detection of $T$. gondii antibodies in animals and meat [17-20]. The National Reference Centre for toxoplasmosis in Reims, France, provided the antigen. Four two-fold dilutions were made starting from $1 / 6$ to $1 / 50$. All results were assessed qualitatively (negative/positive) regardless of dilution.

\section{Statistical analysis}

The overall animal- as well as age class-specific proportions of seropositive animals was calculated, with the associated exact binomial confidence intervals (95\% CI). Because of the relatively small size of the farm sample (60 farms), four risk factors for which exposure was rare (less than 5 rooms or farms) were excluded from the analysis (Table 1). The univariate association between the serological status of pigs (positive/negative) and the independent variables (i.e. the age class and the nine room- and farm-level variables included in the analysis) was analysed using logistic regression models and likelihood ratio tests. Variables for which $P$-values were $\leq 0.20$ were selected for inclusion in the multivariate model. Verifying that variance inflation factor was $<5$ for each of the selected variables checked absence of significant multi-collinearity. A logistic mixed regression model was then used, in which the dependent variable was the pig T. gondii serological status (positive/negative). The selected explicative variables were included as fixed effects, and the farm was included as a random effect. Significance threshold was set to 0.05 .

All statistical analyses were performed using $\mathrm{R}$ [21] (http://www.R-project.org/), using the MASS, lme4 and car packages [22-24].

\section{Results}

Animal, farm and within-farm levels of $T$. gondii frequency of seropositivity

A total number of 3600 sera (60 farms and 60 sera per farm) were taken from the serum bank for serological analysis. Five of these sera gave inconclusive MAT results; hence the analysis was eventually performed on 3595 individual serological results. All herds had at least one seropositive pig, which brought the frequency of seropositive farms at $100 \%(60 / 60)$ (95 \% CI: 94-100 \%). When only one age category was considered, the proportion of seropositive farms (i.e. farms with at least one seropositive animal in the considered category) was $50 \%(18 / 36)$ 
Table 1 Farm-level, room-level and individual-level variables considered as T. gondii seropositivity risk factors in pigs from confined farrow-to-finish farms. Out of risk factors for which exposure is not rare ( $>5$ farms), presence of Danish entry facility, farm size, dairy production, feed distribution mechanism, and age of animals have a statistically significant influence on $T$. gondii seroprevalence in pigs. These factors entered logistic mixed regression model

\begin{tabular}{lll}
\hline Risk factor & $n$ & $\begin{array}{l}\text { Animals - } \\
\text { positive/total (\%) }\end{array}$ \\
\hline $\begin{array}{l}\text { Farm level } \\
\text { Use of specialized } \\
\text { boots }\end{array}$ & 60 farms & \\
$\quad$ No & 20 & $95 / 1198(7.9 \%)$ \\
$\quad$ Yes & 40 & $156 / 2397(6.5 \%)$ \\
$\begin{array}{l}\text { Use of specialized } \\
\text { clothes }\end{array}$ & & \\
No & & \\
Yes & 20 & $92 / 1193(7.7 \%)$ \\
& 40 & $159 / 2402(6.6 \%)$
\end{tabular}

Empty period (days) of farrowing rooms

Empty period (days) of rooms for $>3$ months old animals $\quad 0.30$

Danish entry

No

43

$172 / 2487(6.9 \%)$

Yes

17

$77 / 1048(7.3 \%)$

Number of sows

per farm

Presence of dairy

cattle

No

Yes

207/3236 (6.4\%)

Presence of beef cattle

No

Yes

56

4

Presence of sheep

No

Yes

Presence of goats

No

Yes

Presence of poultry

No

Yes

Room level

Floor type

Slatted floor

Straw

Slatted and concrete floor

Feed distribution
Table 1 Farm-level, room-level and individual-level variables considered as T. gondii seropositivity risk factors in pigs from confined farrow-to-finish farms. Out of risk factors for which exposure is not rare ( $>5$ farms), presence of Danish entry facility, farm size, dairy production, feed distribution mechanism, and age of animals have a statistically significant influence on $T$. gondii seroprevalence in pigs. These factors entered logistic mixed regression model (Continued)

\begin{tabular}{|c|c|c|c|}
\hline Automatic system & 79 & $73 / 1219(5.9 \%)$ & \\
\hline Manual distribution & 155 & 178/2376 (7.5 \%) & \\
\hline Individual level & 3595 pigs & & \\
\hline Age & & & 0.002 \\
\hline$<1$ month & 545 & 29/545 (5.3 \%) & \\
\hline $1-2$ months & 438 & $36 / 438(8.2 \%)$ & \\
\hline 2-3 months & 790 & 76/790 (9.6 \%) & \\
\hline$>3$ months & 1822 & 110/1822 (6.0 \%) & \\
\hline
\end{tabular}

${ }^{a}$ Variables with a low exposure level (less than 5 farms or rooms) were excluded from the multivariate analysis

for suckling piglets ( $<1$ month) (95\% CI: 34-66 \%), $48 \%$ (29/60) for weaned animals (1-2 months old) (95\% CI: 35-61\%), as well as for 2-3 months old fattening pigs (20/42) (95 \% CI: 33-63\%). The proportion of farms with at least one seropositive pig older than 3 months was $82 \%$ (49/60) (95 \% CI: 72-92 \%).

The overall proportion of seropositive animals was 7.0 \% (251/3595; 95 \% CI: 6.2-7.9 \%). It varied according to the age class: $5.3 \%$ in $<1$ month old suckling piglets (29/545; $95 \%$ CI: $3.6-7.6 \%), 8.2 \%$ in $1-2$ months of age weaned animals (36/438; $95 \%$ CI: $5.8-11.1 \%), 9.6 \%$ in pigs $2-3$ months old (76/790; $95 \%$ CI: 7.7-11.9\%), and $6.0 \%$ in animals older than 3 months $(110 / 1822$; 95 \% CI: 5.0-7.2 \%) (Table 1).

The within-herd proportion of seropositive animals ranged between 2 and $28 \%$. This range varied according to the age class: $0-27 \%$ in $<1$ month old suckling piglets, $0-40 \%$ in $1-2$ months of age weaned animals, $0-93 \%$ in pigs $2-3$ months old, and $0-30 \%$ in animals older than 3 months.

\section{Risk factor analysis}

Based on univariate analyses, six variables were selected for inclusion in the multivariate model: the presence of dairy cattle, the herd size (number of sows on a logarithmic scale), the use of specialised clothes inside production units, the feed distribution method, the presence of a Danish entry facility, and the age class (Table 1).

In the multivariate logistic mixed model (Table 2), a significant association between age class and T. gondii seropositivity risk was observed, with increasing oddsratios $(\mathrm{OR})$ for $1-2$ months old weaned piglets $(\mathrm{OR}=$ 1.9, $P=0.04$ ) and for $2-3$ months old fattening pigs $(\mathrm{OR}=2.0, P=0.002)$ when compared to suckling animals 
Table 2 Logistic mixed regression model of T. gondii infection intensity in pigs from farrow-to-finish farms

\begin{tabular}{|c|c|c|c|}
\hline Risk factor & Value & $\begin{array}{l}\text { Odds-ratio } \\
(95 \% \mathrm{Cl})\end{array}$ & $P$-value \\
\hline \multirow[t]{4}{*}{ Age class } & $\begin{array}{l}<1 \text { month - suckling } \\
\text { piglets }\end{array}$ & 1 & \\
\hline & $\begin{array}{l}1-2 \text { months - weaned } \\
\text { piglets }\end{array}$ & $1.9(1.0-3.2)$ & 0.04 \\
\hline & $\begin{array}{l}2-3 \text { months - young } \\
\text { fattening pigs }\end{array}$ & $2.0(1.3-3.2)$ & 0.002 \\
\hline & $\begin{array}{l}>3 \text { months - fattening } \\
\text { pigs }\end{array}$ & ns & 0.29 \\
\hline $\begin{array}{l}\text { Herd size (number } \\
\text { of sows on a } \\
\text { logarithmic scale) }\end{array}$ & $\begin{array}{l}\text { Increase from the } 25 \% \\
\text { (139 sows) to the } 75 \% \\
\text { percentile ( } 251 \text { sows) } \\
\text { of the observed distribution }\end{array}$ & $0.87(0.8-0.9)$ & 0.04 \\
\hline \multirow{2}{*}{$\begin{array}{l}\text { Presence of } \\
\text { dairy cattle }\end{array}$} & No & 1 & \\
\hline & Yes & ns & 0.24 \\
\hline \multirow{2}{*}{$\begin{array}{l}\text { Presence of a } \\
\text { Danish entry facility }\end{array}$} & No & 1 & \\
\hline & Yes & $0.58(0.3-0.9)$ & 0.04 \\
\hline \multirow{2}{*}{$\begin{array}{l}\text { Use of specialised } \\
\text { clothes inside } \\
\text { production units }\end{array}$} & No & 1 & \\
\hline & Yes & ns & 0.77 \\
\hline \multirow{2}{*}{$\begin{array}{l}\text { Feed distribution } \\
\text { method }\end{array}$} & Automatic feeder & 1 & \\
\hline & Manual distribution & ns & 0.38 \\
\hline
\end{tabular}

This model demonstrates an increased seropositivity risk in weaned piglets (1-2 months of age), and 2-3 months old animals as compared to suckling piglets. Seropositivity risk is lower in pigs from larger farms than in animals from small producers. Use of Danish entries on the farm decreases seropositivity risk as well. Presence of dairy production, the use of specialised clothes per room, and methods of feed distribution did not influence $T$. gondii seropositivity of pig bred in confined farms

( $<1$ month of age). However, in $>3$ months old pigs, the seropositivity risk was not significantly different from the one observed in $<1$ month piglets $(P=0.29)$ (Table 2$)$. The number of sows (a quantitative variable, on a logarithmic scale, used as a proxy for herd size) had a significant effect on pig seropositivity risk, with an OR of $0.87(P=0.04)$ for an increase of herd size from the $25 \%$ percentile of its distribution in the sample (139 sows/farm) to the $75 \%$ percentile (251 sows/farm). No significant association was observed between seropositivity risk and the presence of dairy cattle $(P=0.24)$, the feed distribution method $(P=0.38)$, and the use of specialized clothes inside the production units $(P=0.77)$. The presence of a Danish entry facility had a protective effect on pig seropositivity risk with an OR of $0.58(P=0.04)$ (Table 2$)$.

\section{Discussion}

For the last 15 years, surveys of $T$. gondii in pigs raised and consumed throughout Europe were reported from 14 countries, in which various age categories were analysed (piglets, fattening pigs, reproduction animals). In 11 studies sample collection was done at the slaughterhouse, allowing estimation of animal- and national-level prevalence, but not of farm-level and within-farm prevalence. Farm-level studies showed that the major risk factors associated with $T$. gondii infection in pigs were the breeding method, the animal age, the presence of cats, and the absence of rodent control $[5,7,8$, 17, 25-29]. Out of five studies that reported different prevalence between intensive and outdoor farming, four showed significant differences between these two housing types, and only Berger-Schoch et al. [12] reported contradictory results in Switzerland. Studies from Portugal, Spain, France, Serbia, Switzerland, Slovakia, and Germany showed that older age categories, from any housing method (intensive and outdoor), showed higher seroprevalence levels $[7,12,26,29-32]$. As a source of contamination for intensively bred pigs in Europe, studies from Spain and Romania identified cats, and reports from Italy and Spain associated lack of rodent control [26, 27, 33, 34]. At the same time, a study from the Netherlands showed significantly lower $T$. gondii seroprevalence in intensive strictly confined pig farms, without access of cats and rodents into buildings [5]. The same risk factors for T. gondii infection of pigs, age of animals, presence of cats, absence of rodent control, type of farm management, were reported in studies from Brazil and Mexico [35-38]. A study from Paraiba state in Brazil found that feeding with leftovers increases chances for pig infection with T. gondii as well [35]. In the Brazilian state of Toledo, the risk for parasites infection was higher on farms where workers were not assigned to specific area of the farm, other animals had access to pig feeders and drinkers, and where mean piglet number was low [39]. For USA indoor-raised pigs rodent control measures and carcass disposal methods were identified as main risk factors for T. gondii seropositivity [40]. In central China, except for the presence of cats and density of pig population in the pens, the presence of mosquitos and flies, as well as semi-patency pens and low frequency of scavenging represent risk factors associated with T. gondii seropositivity [41].

In the present study, the piglets and fattening pigs from closed farm facilities in France were tested for the presence of anti-T. gondii antibodies. For this purpose, pig sera collected from 2006 to 2008, for respiratory diseases study in farrow-to-finish farms from western France were used. Samples have been kept in sera bank at $-80{ }^{\circ} \mathrm{C}$ without defrosting for the last ten years, which made them valuable for the present analysis, as in previous studies (e.g. [42]). At the same time, for the last ten years biosecurity and hygiene systems in farrow-to-finish farms changed insignificantly, thus the results of this analysis can be applied to nowadays pig producers, as well. This is the first in depth analysis of individual and on farm factors that allows identifying the most vulnerable moments in pig breeding for $T$. gondii infection, 
as well as conditions that favour parasite spread throughout the buildings of farrow-to-finish farms.

The proportion of $100 \%$ positive farms indicates a widespread contamination of pig farms. Animal-level seropositivity rate was relatively high $(7 \%)$ and withinherd seropositivity rate reached $93 \%$ in pigs $2-3$ months old of one of the 60 farms. The animal-level seropositivity rate of $7.0 \%$ reported here is similar to the seroprevalence reported in Serbia, Portugal and Austria, in pigs from same type of confinement [31, 32, 43]. However, the samples analysed in the present study were taken from a previous cross-sectional survey, in which inclusion criteria was the history of respiratory diseases [14], and therefore cannot be considered representative of the French farrow-to-finish farms, although no obvious association exists between the occurrence of respiratory diseases and $T$. gondii (other than hygienic conditions which were explicitly considered in the present study).

The multivariate model of pig seropositivity indicated a significant association between seropositivity risk and age class. Duration of maternal antibodies from naturally infected sows in piglets lasts from three to six weeks of life [44] and in experimental conditions up to 17 weeks [45]. In this period, with the decrease in the concentration of specific $T$. gondii antibodies, young animals become susceptible to infection. The earliest age piglets can get infected orally in experimental conditions is between the fourth and fifth week of life [46]. This goes to show that in piglets younger than one month, a T. gondii seropositive result may be attributed to maternal antibodies. Furthermore, in experimentally infected piglets, newly formed antibodies can be detected only after 2-3 weeks $[46,47]$. Therefore, in the 1-2 months old animals, significantly higher seropositivity risk than in suckling piglets suggests that the seropositive results may in part be due to newly established infections. Furthermore, the higher odds-ratio observed for 2-3 months old animals implies that most probably infection occurs at the end of second month of life, when feeding becomes more diverse. In the crosssectional survey of respiratory diseases upon which the present study is based [14] some pig sera were analysed for infectious agents responsible for alterations of pig's immune system, such as Porcine circovirus (PCV) and Porcine reproductive and respiratory virus (PRRSV). These analyses aimed at documenting viral circulation at the batch level, and the individual serological results were unfortunately not available for the 3595 pig samples used for T. gondii analysis. For this reason, seropositivity for PCV and/or PRRSV could not be considered as risk factors of $T$. gondii seropositivity risk.

Only the study from Serbia showed farm size as a risk factor [31]. Our study shows, as well, that for pigs risk of acquiring $T$. gondii infection was higher in animals living in small pig farms, which may suggest a lower technical and hygienic level in small producers.

The present study shows that installation of a Danish entry facility in the pig breeding farms decreases the $T$. gondii seropositivity risk. In the confined conditions of intensive pig farms, Danish entry is a closed facility with several doors that open successively, one at a time, upon entering and exiting the breeding facility. In the majority of cases it serves for boots and clothes change and sanitation, and prevents direct entering into the buildings. In these conditions even if there are other animal species (potential sources of $T$. gondii infection such as rodents, small birds and cats), their contact with pigs is less likely.

\section{Conclusion}

The described $T$. gondii seropositivity in intensively bred pigs from western France, complements the results of a previous animal-level, nation-wide study, by demonstrating that even in confined conditions of pig breeding, parasite infection exists and that current biosecurity and hygiene measures have not met satisfactory standards for production of Toxoplasma free pork. This is the first in depth analysis of intensive farm conditions and their influence on seropositivity risk. Current study shows that maternal $T$. gondii antibodies are present in suckling piglets, and suggests that the risk of acquiring parasite is the greatest at the end of weaning period. The presence of other livestock productions on the same farm does not appear to have any impact on T. gondii infection. However, small farms where pig breeding is probably not the primary activity, demonstrated increased $T$. gondii levels of seropositivity risk, despite the confined conditions. This study also shows that Danish entry facilities on farm buildings appear to be an effective protection measure from $T$. gondii infection. Additional studies are necessary to focus on specific reservoirs of infection in intensive farms.

\section{Additional file}

Additional file 1: Questionnaire used to collect epidemiological data. (DOCX $23 \mathrm{~kb}$ )

\section{Acknowledgements}

Authors are grateful to the farmers and the related farm organizations for their contribution. They would like also to thank Professor Olin Taft for his help in improving the use of English in the manuscript.

Funding

Not applicable.

Availability of data and material

Sera bank and epidemiological data used in this study are made by Fablet Christelle and Nicolas Rose (ANSES, Unité Epidémiologie et Bien-Etre du Porc; Université Européenne de Bretagne, Laboratoire de Ploufragan-Plouzané, Ploufragan, France) in consent with organisations of pig producers. As such 
materials and serology results can be available upon direct contact of above mentioned co-authors.

\section{Authors' contributions}

VD contributed to conception and design of study, serology analysis, statistical calculations and interpretation of results, drafting the manuscript and revising it critically for important intellectual content. CF contributed to acquisition of sera and epidemiological data, interpretation of results, drafting the manuscript and revising it critically for important intellectual content. RB contributed to serology analysis, revising the manuscript critically for important intellectual content, and has given final approval of the version to be published. NR contributed to conception and design of study, to acquisition of sera and epidemiological data, interpretation of results, revising the manuscript critically for important intellectual content and has given final approval of the version to be published. CP contributed to serology analysis, interpretation of results and revising the manuscript. ODD contributed to revising the manuscript critically for important intellectual content, and has given final approval of the version to be published. PB contributed to conception and design of study, analysis of results, drafting the manuscript, revising it critically for important intellectua content and has given final approval of the version to be published. $\mathrm{BD}$ contributed to statistical analysis and interpretation of data, revising the manuscript critically for important intellectual content, and has given final approval of the version to be published. All authors read and approved the final version of the manuscript.

\section{Competing interests}

The authors declare that they have no competing interests.

\section{Consent for publication}

A written consent from farmers (represented by their organisations o producers) was obtained for the use of pig sera and data in the present study.

\section{Ethics approval and consent to participate}

The study was performed in accordance with current legislation on ethical and welfare recommendations. Anses - Ploufragan collected pig sera in agreement with the obtained license for animal experiment and is registered under the agreement number B-22-745-1 delivered by the official veterinary services.

\section{Author details}

${ }^{1}$ Ecole Nationale Vétérinaire d'Alfort, ANSES, INRA, Université Paris-Est, Laboratoire de santé animale de Maisons-Alfort UMR BIPAR, Maisons-Alfort, France. ${ }^{2}$ ANSES, Unité Epidémiologie et Bien-Etre du Porc, Université Européenne de Bretagne, Laboratoire de Ploufragan-Plouzané, Ploufragan, France. ${ }^{3}$ ANSES, Ecole Nationale Vétérinaire d'Alfort, INRA, Université Paris-Est, Laboratoire de santé animale de Maisons-Alfort, UMR BIPAR, Maisons-Alfort, France. ${ }^{4}$ Institute for Medical Research, National Reference Laboratory for Toxoplasmosis, University of Belgrade, Dr. Subotića 4, P.O. Box 102, Belgrade 11129, Serbia. ${ }^{5}$ ANSES, Université Paris-Est, Laboratoire de santé animale de Maisons-Alfort, Epidemiology unit, Maisons-Alfort, France.

Received: 4 February 2016 Accepted: 12 August 2016

Published online: 24 August 2016

\section{References}

1. Dubey J, Beattie C. Toxoplasmosis in man (homo sapiens). In: Dubey J, Beattie C, editors. Toxoplasmosis of animals and man. Florida: CRC Press, Inc; 1988. p. 41-60.

2. Markovic M, Ivovic V, Stajner T, Djokic V, Klun I, Bobic B, et al. Evidence for genetic diversity of Toxoplasma gondii in selected intermediate hosts in Serbia. Comp Immunol Microbiol Infect Dis. 2014;37(3):173-9.

3. Cook AJ, Gilbert RE, Buffolano W, Zufferey J, Petersen E, Jenum PA, et al. Sources of Toxoplasma infection in pregnant women: European multicentre case-control study. European Research Network on Congenital Toxoplasmosis. BMJ. 2000;321(7254):142-7.

4. Esteban-Redondo I, Maley SW, Thomson K, Nicoll S, Wright S, Buxton D, Innes EA. Detection of $T$. gondii in tissues of sheep and cattle following oral infection. Vet Parasitol. 1999;86(3):155-71.
5. van der Giessen J, Fonville M, Bouwknegt M, Langelaar M, Vollema A Seroprevalence of Trichinella spiralis and Toxoplasma gondii in pigs from different housing systems in The Netherlands. Vet Parasitol. 2007;148(3-4):371-4.

6. Wallander C, Frössling J, Dórea FC, Uggla A, Vågsholm I, Lundén A. Pasture is a risk factor for Toxoplasma gondii infection in fattening pigs. Vet Parasitol. 2016;224:27-32.

7. Djokic V, Blaga R, Aubert D, Durand B, Perret C, Geers R, et al. Toxoplasma gondii infection in pork produced in France. Parasitology. 2016;143(5):557-67.

8. Deksne G, Kirjusina M. Seroprevalence of Toxoplasma gondii in domestic pigs (Sus scrofa domestica) and wild boars (Sus scrofa) in Latvia. J Parasitol. 2013;99(1):44-7.

9. Wallander C, Frossling J, Vagsholm I, Uggla A, Lunden A. Toxoplasma gondii seroprevalence in wild boars (Sus scrofa) in Sweden and evaluation of ELISA test performance. Epidemiol Infect. 2015;143(9):1913-21.

10. Opsteegh M, Swart A, Fonville M, Dekkers L, van der Giessen J. Age-related Toxoplasma gondii seroprevalence in Dutch wild boar inconsistent with lifelong persistence of antibodies. PLoS One. 2011:6(1):e16240.

11. Beral M, Rossi S, Aubert D, Gasqui P, Terrier ME, Klein F, et al. Environmental factors associated with the seroprevalence of Toxoplasma gondii in Wild Boars (Sus scrofa), France. Eco Health. 2012;9(3):303-9.

12. Berger-Schoch AE, Bernet D, Doherr MG, Gottstein B, Frey CF. Toxoplasma gondii in Switzerland: a serosurvey based on meat juice analysis of slaughtered pigs, wild boar, sheep and cattle. Zoonoses Public Health. 2011;58(7):472-8.

13. Dubey JP. Toxoplasmosis in pigs - the last 20 years. Vet Parasitol. 2009;164(2-4): 89-103.

14. Fablet C, Marois-Crehan C, Simon G, Grasland B, Jestin A, Kobisch M, et al. Infectious agents associated with respiratory diseases in 125 farrow-to-finish pig herds: a cross-sectional study. Vet Microbiol. 2012;157(1-2):152-63.

15. Agreste. Les élevages de porcs en France métropolitaine en 2010. Agreste Primeur. 2013;300

16. Halos L, Thebault A, Aubert D, Thomas M, Perret C, Geers R, et al. An innovative survey underlining the significant level of contamination by Toxoplasma gondii of ovine meat consumed in France. Int J Parasitol. 2010; 40(2):193-200

17. Klun I, Djurkovic-Djakovic O, Katic-Radivojevic S, Nikolic A. Cross-sectional survey on Toxoplasma gondii infection in cattle, sheep and pigs in Serbia: seroprevalence and risk factors. Vet Parasitol. 2006;135(2):121-31.

18. Villena I, Durand B, Aubert D, Blaga R, Geers R, Thomas M, et al. New strategy for the survey of Toxoplasma gondii in meat for human consumption. Vet Parasitol. 2012;183(3-4):203-8.

19. Forbes LB, Parker SE, Gajadhar AA. Performance of commercial ELISA and agglutination test kits for the detection of anti-Toxoplasma gondii antibodies in serum and muscle fluid of swine infected with 100,300, 500 or 1000 oocysts. Vet Parasitol. 2012;190(3-4):362-7.

20. Pardini L, Maksimov P, Herrmann DC, Bacigalupe D, Rambeaud M, Machuca M, More G, Basso W, Schares G, Venturini MC. Evaluation of an in-house TgSAG1 (P30) lgG ELISA for diagnosis of naturally acquired Toxoplasma gondii infection in pigs. Vet Parasitol. 2012;189(2-4):204-10.

21. R development Core Team. R: A Language and Environment for Statistical Computing. Vienna: R Foundation for Statistical Computing; 2010.

22. Venables WN, Ripley BD. Modern Applied Statistics with S, vol. 4. New York: Springer; 2002.

23. Bates D, Maechler M, Bolker BM, Walker S. Lme4: Linear mixed-effects models using Eigen and S4. 2014.

24. Fox J, Weisberg S. An (R) Copanion to Applied Regression. 2nd ed. Thousand Oaks: Sage; 2011.

25. Garcia-Bocanegra I, Dubey JP, Simon-Grife M, Cabezon O, Casal J, Allepuz A, et al. Seroprevalence and risk factors associated with Toxoplasma gondii infection in pig farms from Catalonia, north-eastern Spain. Res Vet Sci. 2010;89(1):85-7.

26. Garcia-Bocanegra I, Simon-Grife M, Dubey JP, Casal J, Martin GE, Cabezon O, et al. Seroprevalence and risk factors associated with Toxoplasma gondii in domestic pigs from Spain. Parasitol Int. 2010;59(3):421-6.

27. Paștiu Al, Györke A, Blaga R, Mircean V, Rosenthal BM, Cozma V. In Romania, exposure to Toxoplasma gondii occurs twice as often in swine raised for familial consumption as in hunted wild boar, but occurs rarely, if ever, among fattening pigs raised in confinement. Parasitol Res. 2013;112(6):2403-7.

28. Berger-Schoch AE, Herrmann DC, Schares G, Müller N, Bernet D, Gottstein B, Frey CF. Prevalence and genotypes of Toxoplasma gondii in feline faeces (oocysts) and meat from sheep, cattle and pigs in Switzerland. Vet Parasitol. 2011;177(3-4):290-7. 
29. Damriyasa IM, Bauer C, Edelhofer R, Failing K, Lind P, Petersen E, Schares G, Tenter AM, Volmer R, Zahner H. Cross-sectional survey in pig breeding farms in Hesse, Germany: seroprevalence and risk factors of infections with Toxoplasma gondii, Sarcocystis spp. and Neospora caninum in sows. Vet Parasitol. 2004;126(3):271-86.

30. Turčeková L', Antolová D, Reiterová K, Spišák F. Occurrence and genetic characterization of Toxoplasma gondii in naturally infected pigs. Acta Parasitol. 2013;58(3):361-6.

31. Klun IV M, Yera H, Nikolic A, Ivovic V, Bobic B, Bradonjic S, et al. Toxoplasma gondii infection in slaughter pigs in Serbia: seroprevalence and demonstration of parasites in blood. Vet Res. 2011:42(1):17.

32. Lopes AP, Dubey JP, Neto F, Rodrigues A, Martins T, Rodrigues M, et al. Seroprevalence of Toxoplasma gondii infection in cattle, sheep, goats and pigs from the North of Portugal for human consumption. Vet Parasitol. 2013:193(1-3):266-9.

33. Cabezon O, Garcia-Bocanegra I, Molina-Lopez R, Marco I, Blanco JM, Hofle U, et al. Seropositivity and risk factors associated with Toxoplasma gondii infection in wild birds from Spain. PLoS One. 2011;6(12):e29549.

34. Veronesi F, Ranucci D, Branciari R, Miraglia D, Mammoli R, Fioretti DP. Seroprevalence and risk factors for Toxoplasma gondii infection on finishing swine reared in the Umbria region, central Italy. Zoonoses Public Health. 2011:58(3):178-84.

35. Feitosa TF, Vilela VL, de Melo LR, de Almeida Neto JL, Souto DV, de Morais DF, et al. Toxoplasma gondii and Neospora caninum in slaughtered pigs from Northeast, Brazil. Vet Parasitol. 2014;202(3-4):305-9.

36. de Sousa RA, Lemos Jda F, Farias LA, Lopes CD, dos Santos KR. Seroprevalence and risk factors for Toxoplasma gondii infection in pigs in southern Piaui. Rev Bras Parasitol Vet. 2014;23(1):98-100.

37. Luciano DM, Menezes RC, Ferreira LC, Nicolau JL, Das Neves LB, Luciano RM, et al. Occurrence of anti-Toxoplasma gondii antibodies in cattle and pigs slaughtered, State of Rio de Janeiro. Rev Bras Parasitol Vet. 2011;20(4):351-3.

38. Alvarado-Esquivel C, Estrada-Malacon MA, Reyes-Hernandez SO, PerezRamirez JA, Trujillo-Lopez JI, Villena I, Dubey JP. High prevalence of Toxoplasma gondii antibodies in domestic pigs in Oaxaca State, Mexico. J Parasitol. 2012;98(6):1248-50

39. Piassa FR, de Araújo JB, da Rosa RC, Mattei RJ, da Silva RC, Langoni H, da Silva AV. Prevalence and risk factors for Toxoplasma gondii infection in certified and non-certified pig breeding farms in the Toledo microregion, PR, Brazil. Rev Bras Parasitol Vet. 2010;19(3):152-6.

40. Hill DE, Haley C, Wagner B, Gamble HR, Dubey JP. Seroprevalence of and risk factors for Toxoplasma gondii in the US swine herd using sera collected during the National Animal Health Monitoring Survey (Swine 2006). Zoonoses Public Health. 2010;57(1):53-9.

41. Tao Q, Wang Z, Feng H, Fang R, Nie H, Hu M, Zhou Y, Zhao J. Seroprevalence and risk factors for Toxoplasma gondii infection on pig farms in central China. J Parasitol. 2011:97(2):262-4.

42. Magar R, Muller P, Larochelle R. Retrospective serological survey of antibodies to porcine circovirus type 1 and type 2. Can J Vet Res. 2000;64(3):184-6.

43. Steinparzer R, Reisp K, Grunberger B, Kofer J, Schmoll F, Sattler T. Comparison of different commercial serological tests for the detection of Toxoplasma gondii antibodies in serum of naturally exposed pigs. Zoonoses Public Health. 2015;62(2):119-24.

44. Garcia-Bocanegra I, Simon-Grife M, Sibila M, Dubey JP, Cabezon O, Martin G, Almeria S. Duration of maternally derived antibodies in Toxoplasma gondii naturally infected piglets. Vet Parasitol. 2010;170(1-2):134-6.

45. Dubey JP, Urban Jr JF. Diagnosis of transplacentally induced toxoplasmosis in pigs. Am J Vet Res. 1990;51(8):1295-9.

46. Jurankova J, Basso W, Neumayerova H, Balaz V, Janova E, Sidler X, et al. Brain is the predilection site of Toxoplasma gondii in experimentally inoculated pigs as revealed by magnetic capture and real-time PCR. Food Microbiol. 2014;38:167-70.

47. Lind P, Haugegaard J, Wingstrand A, Henriksen SA. The time course of the specific antibody response by various ELISAs in pigs experimentally infected with Toxoplasma gondii. Vet Parasitol. 1997;71(1):1-15.

\section{Submit your next manuscript to BioMed Central and we will help you at every step:}

- We accept pre-submission inquiries

- Our selector tool helps you to find the most relevant journal

- We provide round the clock customer support

- Convenient online submission

- Thorough peer review

- Inclusion in PubMed and all major indexing services

- Maximum visibility for your research

Submit your manuscript at www.biomedcentral.com/submit
Biomed Central 